\title{
RvD1 accelerates the resolution of inflammation by promoting apoptosis of the recruited macrophages via the ALX/FasL-FasR/ caspase-3 signaling pathway
}

\author{
Shu-yang Xiang ${ }^{1}$, Yang $\mathrm{Ye}^{1}$, Qian Yang ${ }^{1}$, Hao- ran Xu ${ }^{1}$, Chen-xi Shen ${ }^{1}$, Min-qi Ma ${ }^{1}$, Shao-wu Jin ${ }^{1}$, Hong-xia Mei ${ }^{1}$, Sheng-xing Zheng ${ }^{1}$,
} Fang-gao Smith ${ }^{1,2}$, Sheng-wei Jin (iD) ${ }^{1 凶}$ and Qian Wang $\mathbb{D i D}^{1 凶}$

(C) The Author(s) 2021

The uncontrolled inflammatory response caused by a disorder in inflammation resolution is one of the reasons for acute respiratory distress syndrome (ARDS). The macrophage pool markedly expands when inflammatory monocytes, known as recruited macrophages, migrate from the circulation to the lung. The persistent presence of recruited macrophages leads to chronic inflammation in the resolution phase of inflammation. On the contrary, elimination of the recruited macrophages at the injury site leads to the rapid resolution of inflammation. Resolvin D1 (RvD1) is an endogenous lipid mediator derived from docosahexaenoic acid. Mice were administered RvD1 via the tail vein 3 and 4 days after stimulation with lipopolysaccharide. RvD1 reduced the levels of the inflammatory factors in the lung tissue, promoted the anti-inflammatory M2 phenotype, and enhanced the phagocytic function of recruited macrophages to alleviate acute lung injury. We also found that the number of macrophages was decreased in BAL fluid after treatment with RvD1. RvD1 increased the apoptosis of recruited macrophages partly via the FasL-FasR/caspase3 signaling pathway, and this effect could be blocked by Boc-2, an ALX/PRP2 inhibitor. Taken together, our findings reinforce the concept of therapeutic targeting leading to the apoptosis of recruited macrophages. Thus, RvD1 may provide a new therapy for the resolution of ARDS.

Cell Death Discovery (2021)7:339; https://doi.org/10.1038/s41420-021-00708-5

\section{INTRODUCTION}

Acute respiratory distress syndrome (ARDS) is a common critical condition that results in high mortality [1]. Its pathophysiological mechanism manifests as a severe inflammatory response that is characterized by the infiltration of large inflammatory cells and cytokine production [2]. Recruited macrophages, derived from the mononuclear cells of the bone marrow, accumulate in the lungs following an injury [3-5]. The persistent presence of recruited macrophages at the injury site, which mainly constitutes the proinflammatory M1 phenotype, leads to chronic inflammation [5]. Therefore, removal of the excessive recruited macrophages is important to expedite the alleviation of inflammation and protect the lung tissue.

Several molecules that play a role in alleviating inflammation have been elucidated and named as special anti-inflammatory mediators (SPM). These molecules exert anti-inflammatory and anti-infective effects [6]. Resolvin D1 (RvD1) is an endogenous lipid mediator derived from docosahexaenoic acid (DHA), which has been proven to exert anti-inflammatory effects in several disease models [7]. RvD1 limits the infiltration of neutrophils during the subsided phase of inflammation, regulates the migration of human polymorphonuclear leukocyte-endothelial cells [8], inhibits the release of proinflammatory cytokines [9], and enhances phagocytic function and cell apoptosis [10]. During acute lung injury, macrophages are divided into resident and recruiting macrophages [11]. Resident macrophages have a certain degree of self-maintenance in a stable state [12] and are the key coordinators of inflammation and repair in the lungs [13]. The recruitment of macrophages stems from monocytes circulating in the blood. Monocyte chemotactic protein (MCP)-1 promotes the recruitment of monocytes to inflammatory tissues to perform their functions [14]. These two cell types play different functions in acute lung injury. Studies show that the depletion and recruitment of macrophages can reduce acute allergic lung inflammation [15]. Elimination of macrophages from chronic injury sites results in the rapid alleviation of inflammation [16, 17]. Additionally, the recruited macrophages undergo apoptosis during inflammation [18] and are subsequently cleared by the neighboring phagocytes, which also constitutes a necessary step in the resolution of acute lung inflammation [19].

In this study, we determined the effect of RvD1 on the recruitment of macrophages during the resolution phase of lipopolysaccharide (LPS)-induced inflammation. We found that RvD1 administered at the peak of inflammation enhanced efferocytosis and M2 phenotype in the recruited macrophages and reduced the production of inflammatory cytokines. Moreover, RvD1 could remove the recruited macrophages by accelerating ALX/Fas ligand (FasL)-FasR/caspase-3-mediated apoptosis and rapidly promoted the resolution of inflammation.

\footnotetext{
${ }^{1}$ Department of Anesthesia and Critical Care, The Second Affiliated Hospital and Yuying Children's Hospital of Wenzhou Medical University, Zhejiang 325027, China. ${ }^{2}$ nstitute of
}

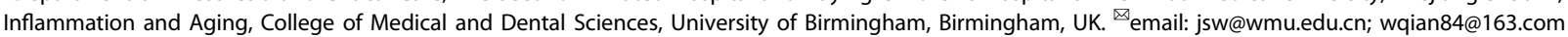




\section{RESULTS}

RvD1 attenuates LPS-induced lung injury to promote resolution of inflammation

After the intratracheal administration of LPS $(1 \mathrm{mg} / \mathrm{kg})$, the number of neutrophils in the BALF increased sharply, peaked on the third day, and then decreased to a steady state by day 8 (Fig. 1A). The body weights of mice showed a decreasing trend and reached the minimum on the third day (Fig. 1B). Therefore, the third day was the peak of inflammation, and after this day, a resolution phase was observed. Mice were administered RvD1 on the third day. After $24 \mathrm{~h}$, the weight of mice in the LPS + RvD1 group increased compared to those in the LPS group $(P<0.05)$ (Fig. 1B). Moreover, the general condition of mice improved, and the appetite of mice in the RvD1 treatment group increased.

The lung tissues of mice in the LPS group were seriously damaged. RvD1 treatment alleviated lung tissue damage (Fig. 1C). The lung injury score was significantly reduced (S1). Furthermore, levels of the inflammatory factors TNF- $\alpha$, IL-1 $\beta$, and IL- 6 in the lung tissue homogenate were decreased in mice in the LPS + RvD1 group compared with those in the LPS group (Fig. 1D). However, there were no significant differences in the levels of these markers between the CTR and RvD1 groups.

The number of neutrophils decreased and was one of the important indicators of the resolution of inflammation. On the fifth day, the F4/80-LY6C + neutrophils in the BALF decreased in the LPS + RvD1 group compared with the LPS group $(P<0.05)$ (Fig. 1E).

\section{RvD1 reduces levels of the recruited macrophages in the resolution phase}

The recruited macrophages were identified as F4/80 + LY6C + (Fig. 2A). The number and percentage of recruited macrophages in the alveolar lavage fluid decreased in the LPS + RvD1 group compared with the LPS group (Fig. 2B). The phagocytosis of the recruited macrophages was determined. The mean fluorescence intensity (MFI) of FITC-LPS on the recruited macrophages in the RvD1 treatment group was higher than that in the LPS group (Fig. 2C). Moreover, treatment with RvD1 reduced the expression of CD86 and iNOS levels (Fig. 2F) and increased CD206 levels in the recruited macrophages (Fig. 2G).

\section{RvD1 increases the numbers of the recruited macrophages in the lymph nodes}

Next, we attempted to elucidate why the levels of the recruited macrophages decreased after treatment with RvD1. The pulmonary lymph node homogenate was used to assess the number of recruited macrophages using flow cytometry. As shown in Fig. 3A, $B$, the number and percentage of the recruited macrophages in the homogenate were increased in the LPS + RvD1 group compared with the LPS group.

\section{RvD1 promotes apoptosis of recruited macrophages in LPS- induced lung injury in vivo}

Next, the apoptosis and proliferation in the recruited macrophages were measured using flow cytometry. As shown in Fig. $4 \mathrm{~A}-\mathrm{C}$, treatment with RvD1 promoted apoptosis of the recruited macrophages; however, there were no effects on proliferation.

Levels of the apoptosis-related proteins were determined using flow cytometry and RT-PCR. Levels of the pro-apoptosis protein, Fas, were increased in the LPS + RvD1 group compared with the LPS group in the recruited macrophages ( $p=0.0377$ ) (Fig. 4D). The FasL in $C D 8^{+}$T cells was much higher in the LPS + RvD1 group compared with the LPS group $(p=0.0041)$ (Fig. 4E). Treatment with Fas-blocking antibody (LPS + RvD1+FasL blocking Ab group) reduced the cell apoptosis compared with the LPS + RvD1 group $(p=0.0011)$ (Fig. 4F). Bax was decreased in the LPS + RvD1 group compared with the LPS group in the recruited macrophages ( $p=$ 0.0170) (Fig. 4G). To further elucidate the mechanism of macrophage apoptosis, the recruited macrophages were sorted to extract RNA. Fas expression was increased, Bax gene expression was unchanged, and intracellular $\mathrm{Mcl}-1$ gene expression was decreased in the LPS + RvD1 group compared to that in the LPS group (Fig. $4 \mathrm{H}-J$ ).

\section{Protective effects of RvD1 on LPS-induced BMDMs in vitro} LPS induced the apoptosis of macrophages in a time- and dosedependent manner. Treatment with $1 \mu \mathrm{g} / \mathrm{mL}$ of LPS resulted in the apoptosis of macrophages, which peaked at 6-12 h (Fig. 5A). Different concentrations of LPS, including $1,10,100$, and $1000 \mathrm{ng} /$ $\mathrm{mL}$, were used in this study. We found that $1000 \mathrm{ng} / \mathrm{mL}$ LPS led to the maximum level of apoptosis of the macrophages, and this concentration was used in subsequent experiments (Fig. 5B, C).

iNOS levels were detected using western blotting, and the protein expression of iNOS reached its peak at $12 \mathrm{~h}$ and then decreased (Fig. 5D). At the same time, CD86 levels also peaked at $12 \mathrm{~h}$ (Fig. 5E). Therefore, in this study, cells were stimulated with LPS for $12 \mathrm{~h}$ in the presence or absence of RvD1.

As shown in Fig. 5F, G, the increased expression of iNOS and CD86 was abolished by treatment with RvD1. Moreover, treatment with RvD1 also promoted the phagocytosis of fluorescent LPS by the macrophages (Fig. $5 \mathrm{H}$ ).

\section{RvD1 promotes apoptosis of BMDMs through Fas}

The primary cells were cultured with LPS and/or RvD1 for $12 \mathrm{~h}$ and the extent of apoptosis was determined using flow cytometry. As shown in Fig. 6A, treatment with RvD1 enhanced apoptosis ( $p=$ 0.0006). Compared to that in the LPS group, the caspase 8 activity was increased in the LPS + RvD1 group $(p=0.0406)$. Compared to that in the CTR group, the protein expression of cleaved caspase- 3 and cleaved PARP was increased in the LPS group. This increase in protein expression was abrogated by treatment with RvD1 (Fig. 6C, D). Meanwhile, the mRNA expression of Fas was increased in the LPS + RvD1 group compared with the LPS group (Fig. 6E). However, there were no significant differences in the mRNA expression of Bax and $\mathrm{Mcl}-1$ between the LPS and LPS + RvD1 groups (Fig. 6F, G). Moreover, there were no changes in the level of $\mathrm{Bax} / \mathrm{BCl}-2$ protein between the LPS and LPS + RvD1 groups (Fig. 6H).

\section{The protective effect of RvD1 on lung tissues is dependent on ALX/PRP2}

The ALX/PRP2 inhibitor, BOC-2, was administered $1 \mathrm{~h}$ before the RvD1 injection. As seen in Fig. 7A, the protective effect of RvD1 on lung tissues was abolished by BOC-2. RvD1-induced neutrophil infiltration was abrogated by BOC-2 (Fig. 7B), whereas RvD1induced apoptosis of the recruited macrophages (Fig. 7C), Fas expression (Fig. 7D) of the recruited macrophages, and FasL expression on $\mathrm{CD}^{+} \mathrm{T}$ cells (Fig. 7E) was abrogated by BOC-2.

\section{DISCUSSION}

ARDS is a common cause of respiratory failure in critically ill patients. Patients with ARDS who need intensive care have a mortality rate of $30-40 \%$ [20]. ARDS is characterized by the infiltration of excessive inflammatory cells. Thus, the removal of these cells could resolve inflammation. In our study, we found that neutrophils in the alveolar lavage fluid markedly increased in the first three days, subsequently decreased slowly, and then entered the phase of inflammation resolution. We found that treatment with RvD1 during the peak of inflammation restored the body weights of the mice, improved the pathophysiological changes in their lungs, decreased TNF- $a, I L-1 \beta$, and IL- 6 levels in the lung tissue homogenate, and reduced neutrophil counts in the BALF. However, it was worth noting that normal mice were unaffected. Together, these results indicated that RvD1 could protect the lung 
A

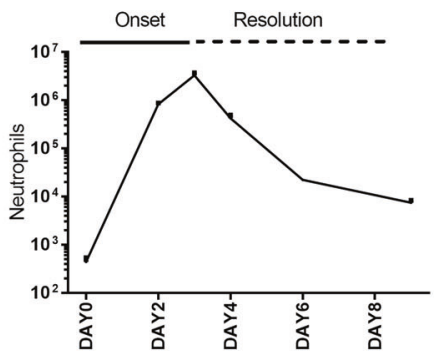

B

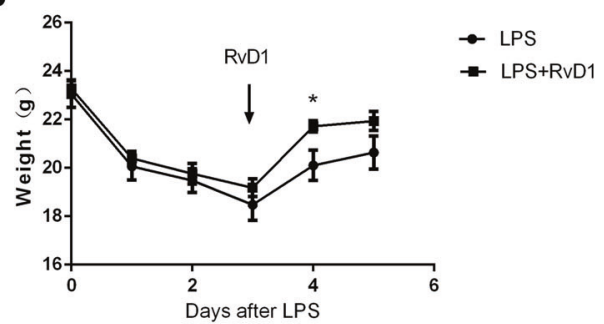

C

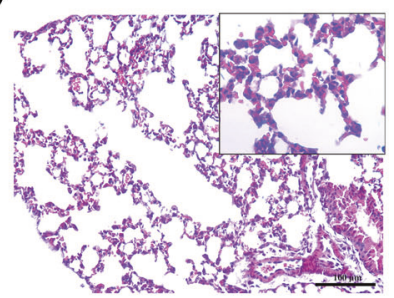

CTR

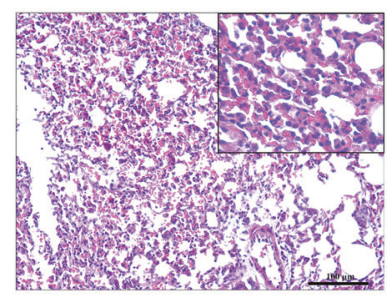

LPS

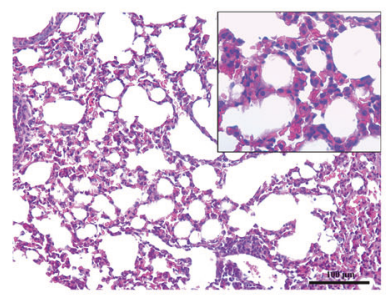

LPS+RvD1

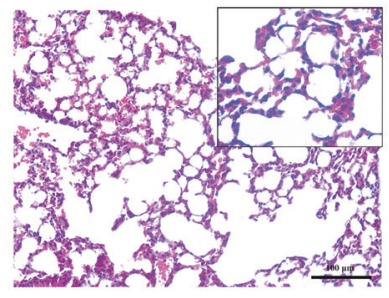

RvD1

D
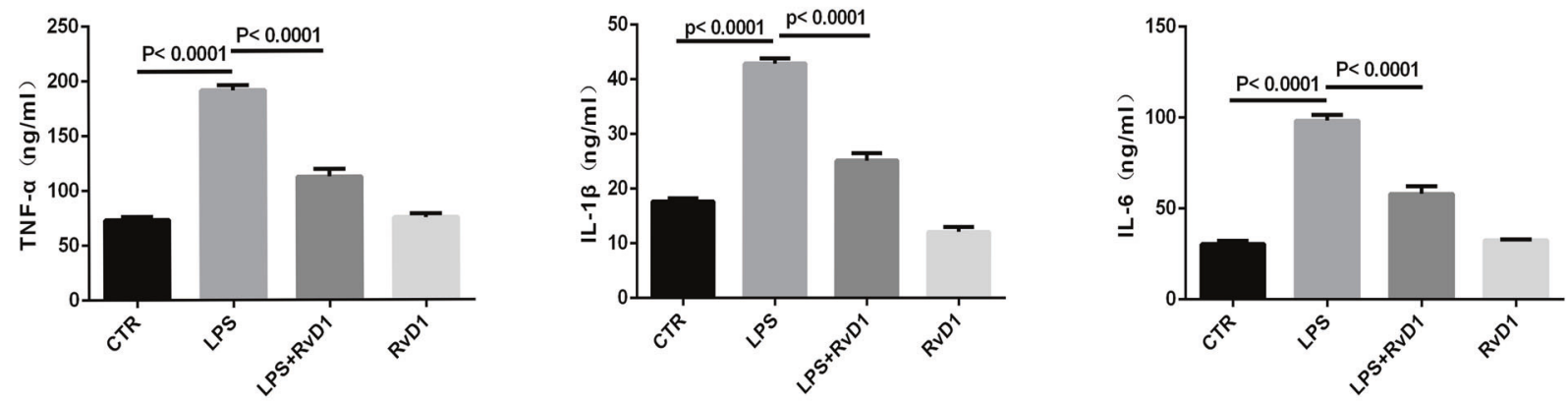

E
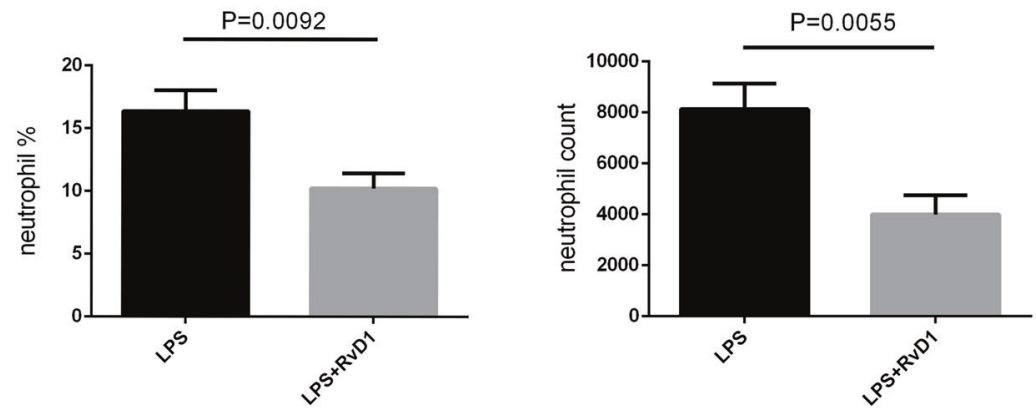

Fig. 1 RvD1 attenuates LPS-induced lung injury to promote resolution of inflammation. A After LPS stimulation, neutrophil counts in the BALF were detected using flow cytometry. B RvD1 was administered through the tail vein on day 3 and the weights of mice were determined. C Treatment with RvD1 protected the lung tissues in LPS-induced ARDS. D Aerosol inhalation of LPS significantly increased TNF- $\alpha$, IL-1 $\beta$, and IL-6 levels in lung tissue, and RvD1 treatment decreased the levels of these inflammatory factors. E Treatment with RvD1 reduced infiltration of neutrophils in the BALF.

tissues of mice with ARDS during the resolution phase of inflammation.

A large number of neutrophils and recruited macrophages enter and accumulate in the lung after lung injury. The persistence of macrophages is a sign of chronic inflammation and can sometimes lead to fibrosis [16, 17]. Our findings suggested that treatment with RvD1 reduced the number of recruited macrophages, inhibited the expression of the recruited macrophages with the M1 phenotype, upregulated $M 2$ expression, and promoted phagocytosis in vivo. In our in vitro study, we found that the expression of iNOS and CD86 after LPS stimulation was time dependent; it peaked at $12 \mathrm{~h}$ and then declined. Therefore, we detected the effect of RvD1 on BMDMs at $12 \mathrm{~h}$. RvD1 suppressed the expression of iNOS and CD86 and promoted phagocytosis. Consistent with our results, a previous study has reported that RvD1 induces the polarization of macrophages and enhances phagocytosis [21, 22]. Our findings indicated that RvD1 could downregulate the recruited macrophage M1 phenotype, promote phagocytosis, and accelerate their removal to promote the resolution of inflammation. It is interesting to assess the presence and function of the recruited macrophages during the resolution phase of inflammation. Some recruited macrophages may migrate from tissues through the lymph and are capable of exiting the tissues to recirculate. We found that RvD1 increased 


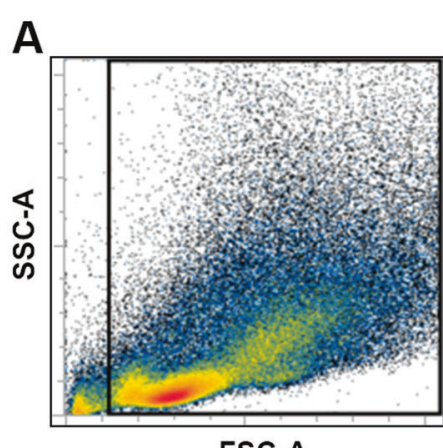

B

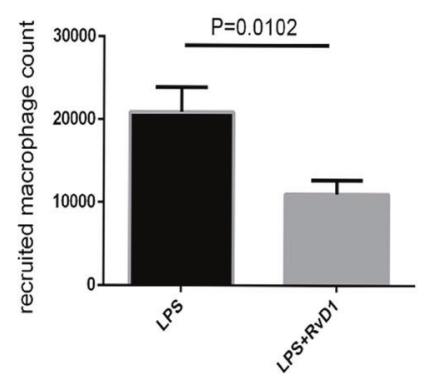

D

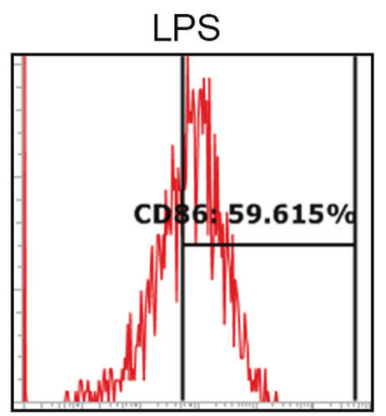

E

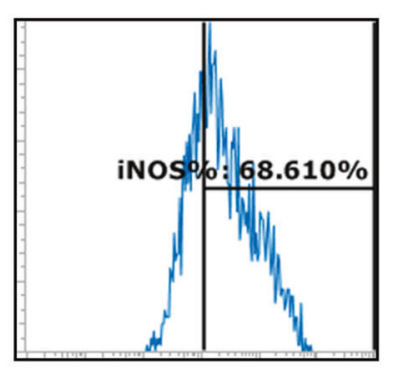

$\mathbf{F}$

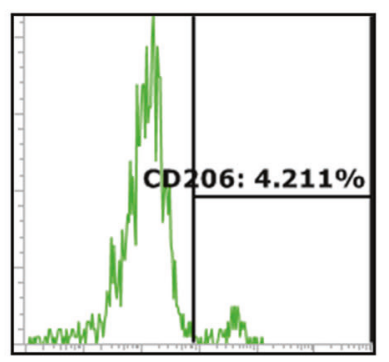

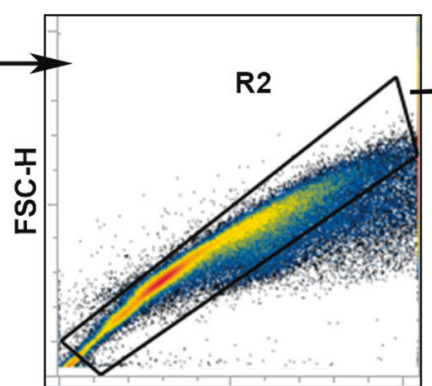

FSC-A
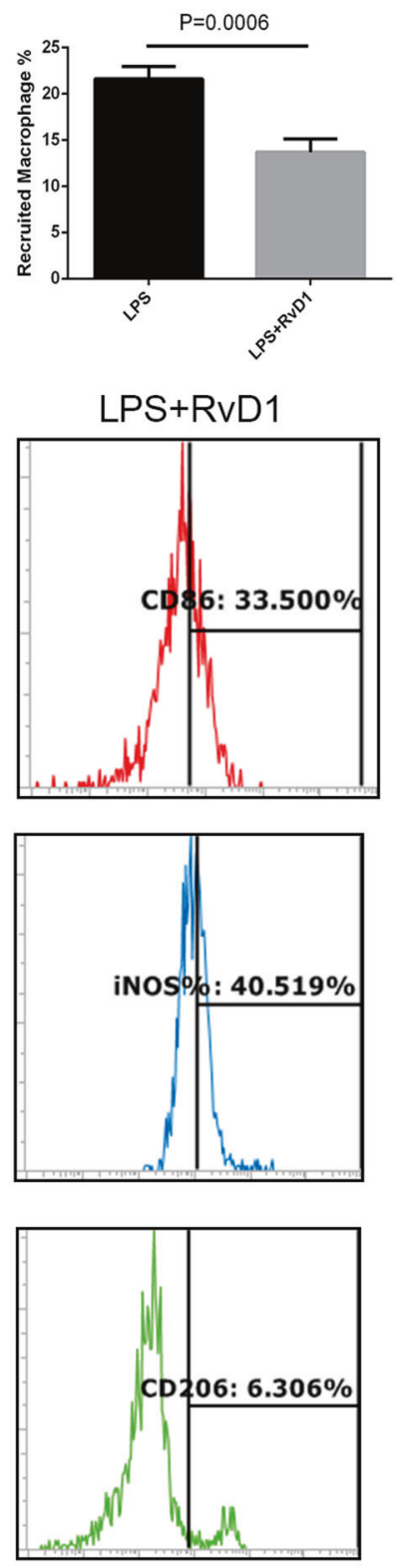

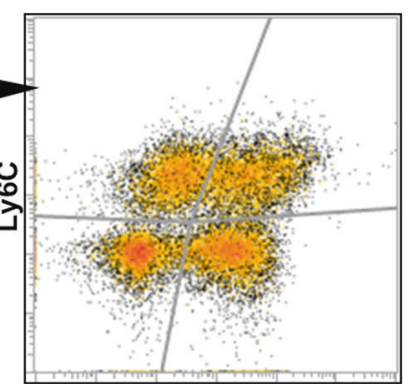

F4/80

C
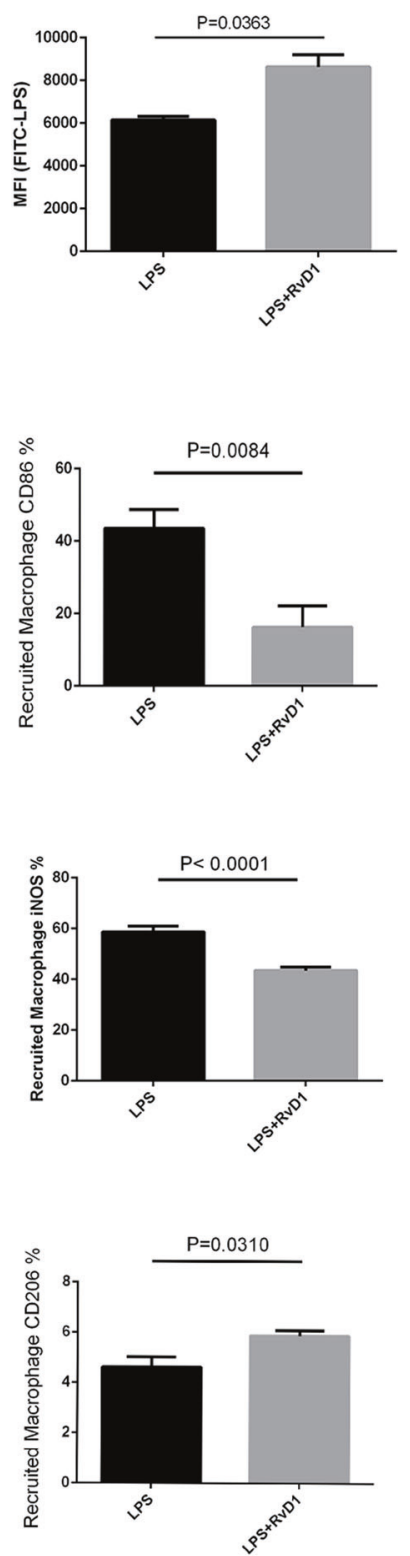

Fig. 2 RvD1 upregulates the recruited macrophages M2 phenotype, enhances phagocytic function, and reduces their accumulation in vivo. A Distinguished the recruited macrophages with F4/80 + LY6C +. B Treatment with RvD1 reduced the number of recruited macrophages in the BALF. C Determination of fluorescence intensity of LPS in the recruited macrophages from BALF after $12 \mathrm{~h}$. Expression of CD86 (D), iNOS (E), and CD206 (F) in BALF from mice were measured in recruited macrophages in LPS with or without RvD1 administration. 

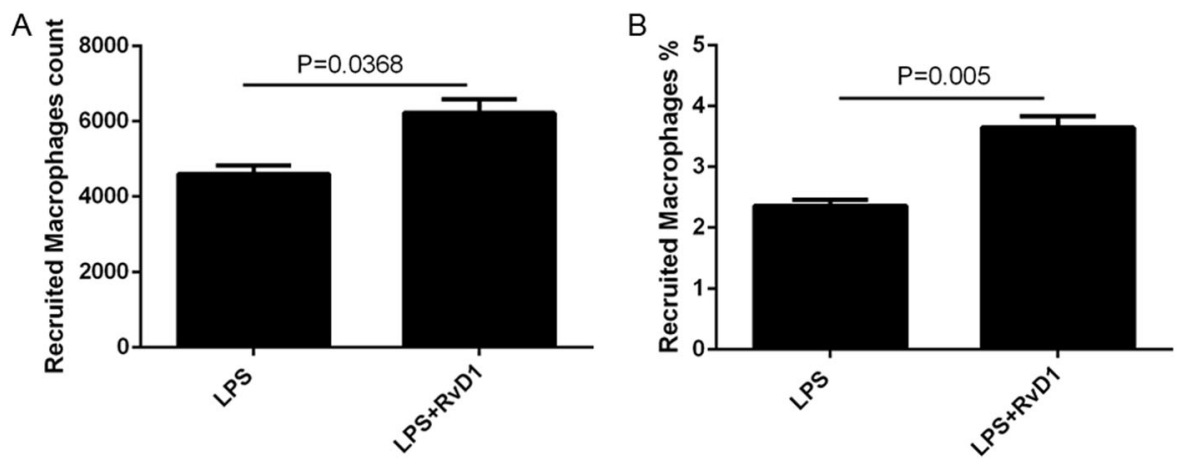

Fig. 3 RvD1 increases the numbers of recruited macrophages in the lymph nodes. The numbers (A) and percentage (B) of the recruited macrophages in the pulmonary lymph node homogenate were detected using flow cytometry.

A

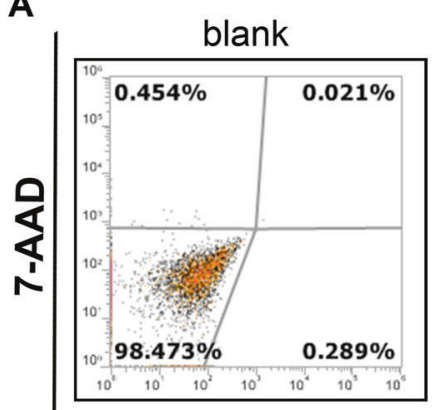

LPS

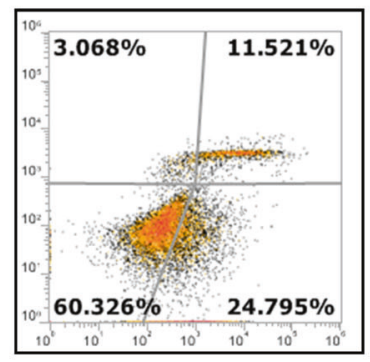

LPS+RvD1

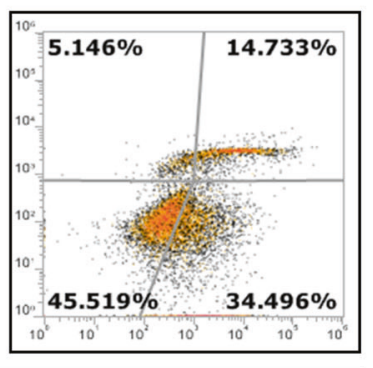

B

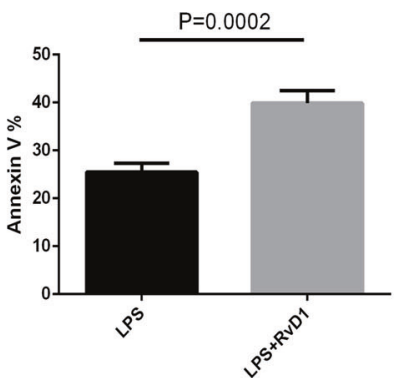

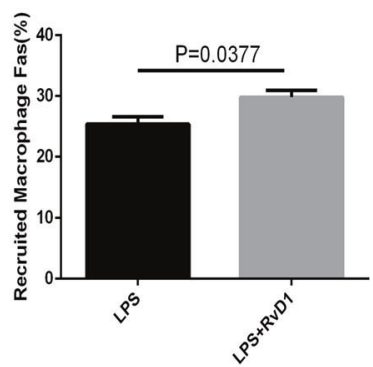

H

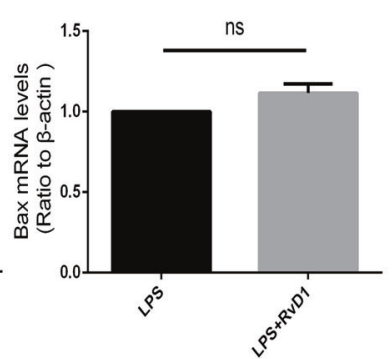

$\mathbf{F}$

E

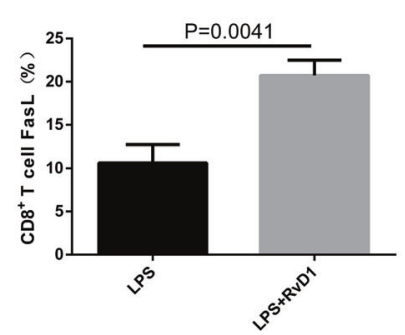

I

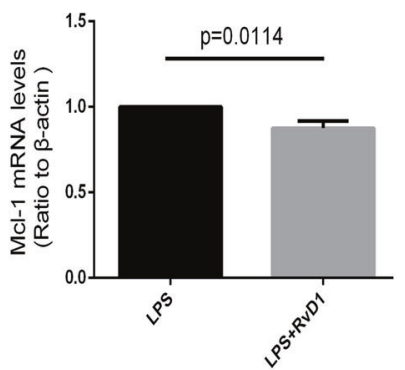

$\mathbf{J}$
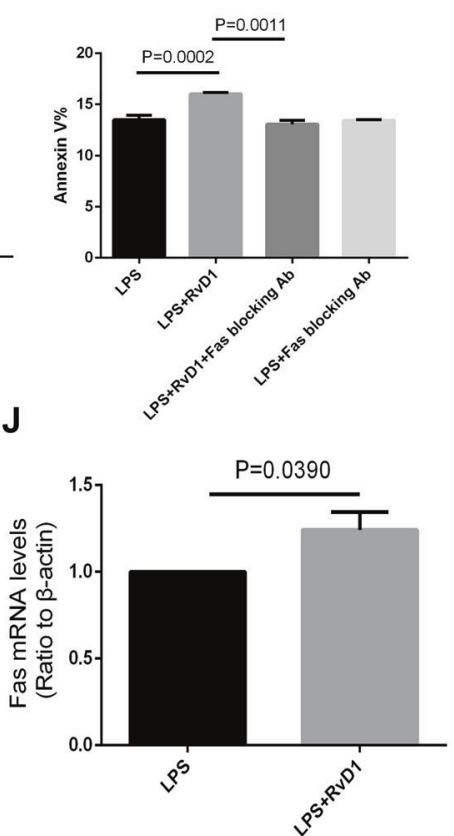

Fig. 4 RvD1 promotes apoptosis of recruited macrophages in LPS-induced lung injury in vivo. A Staining clusters of Annexin V and 7-AAD on recruited macrophages. B Percentage of cells expressing Annexin $V^{+} 7-A A^{-}$on recruited macrophages. C Effect of RvD1 on the proliferation of recruited macrophages. D Fas levels (\%) on the recruited macrophages. E FasL levels (\%) on CD8 ${ }^{+} \mathbf{T}$ cells. F The effect of Fasblocking antibody on the apoptosis of recruited macrophage. (G) Bax expression (\%) on recruited macrophages. Expression of Bax (H), Mcl-2 (I), Fas (J) mRNA in the recruited macrophages. 
A

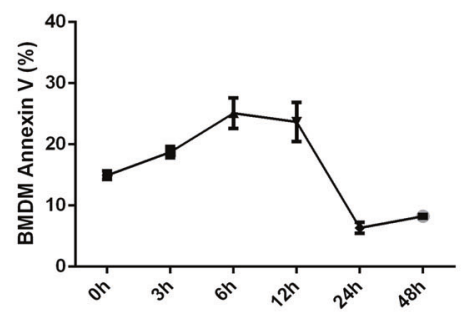

D

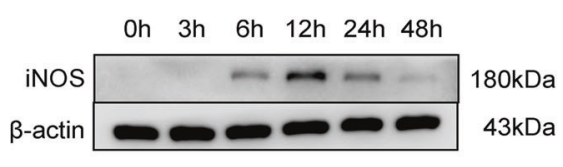

F

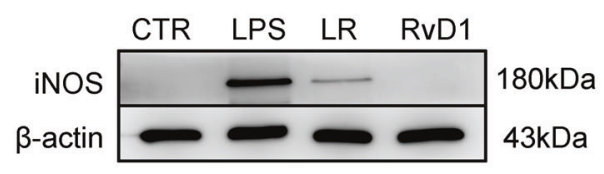

H

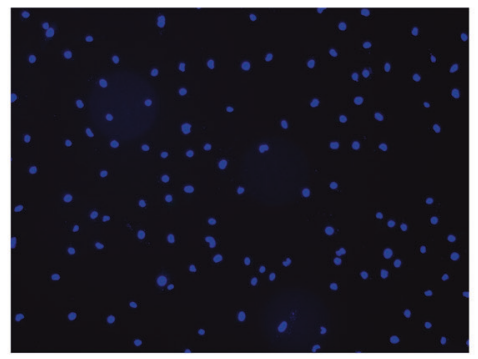

CTR
B
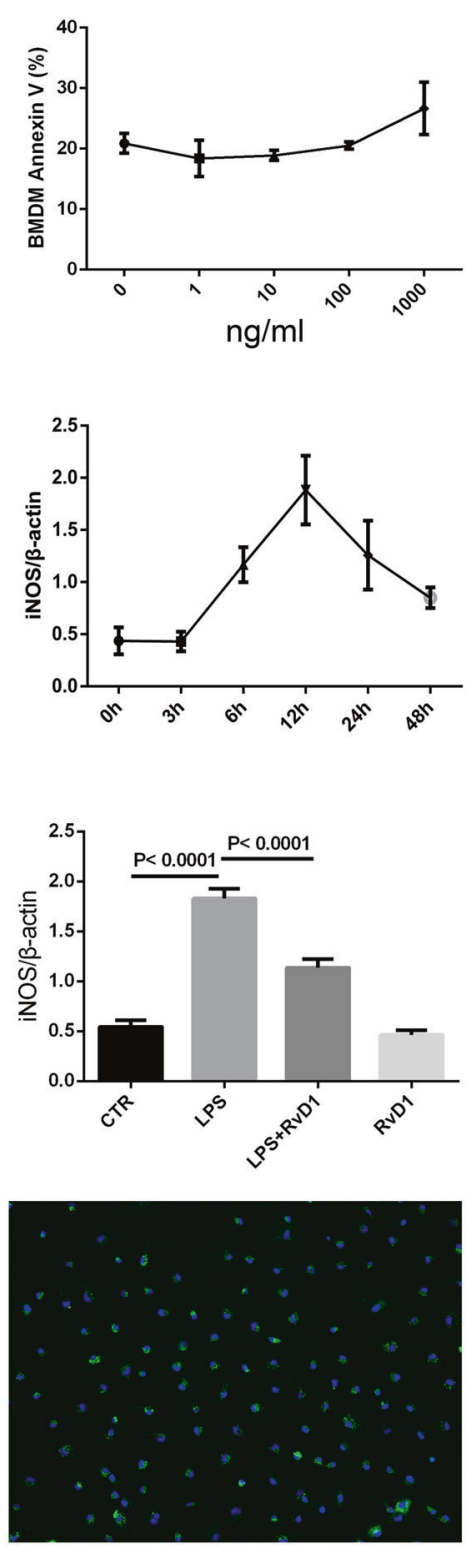

LPS
C

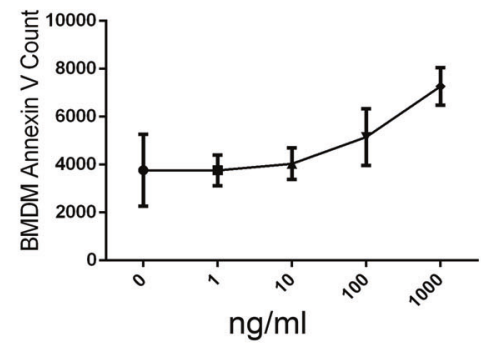

E

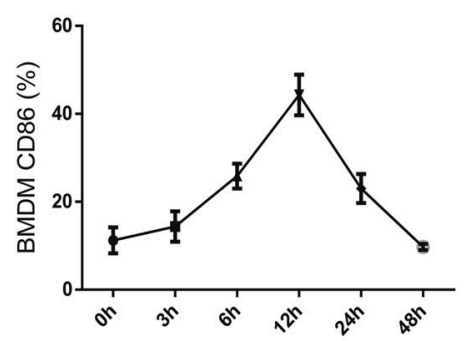

G
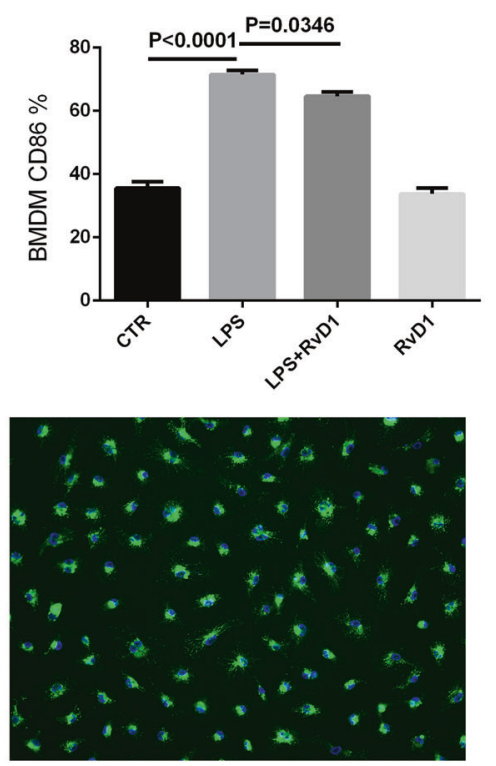

LPS+RvD1

Fig. 5 Protective effects of RvD1 on LPS-induced BMDMs in vitro. A Time-course of LPS-induced apoptosis. About $10^{6}$ macrophages were treated with $1 \mu \mathrm{g} / \mathrm{mL}$ LPS at the indicated time. B) Cells were treated for $12 \mathrm{~h}$ with the indicated. concentrations of LPS; C Annexin V was detected as indicated above. D. Expression of LPS-induced iNOS was measured using western blotting. E LPS induces the expression of CD86. F Western blotting was used to determine the expression of iNOS in BMDMs treated with $1 \mu \mathrm{g} / \mathrm{mL}$ of LPS in the presence or absence of 100 nM RvD1. G RvD1 inhibits LPS-induced CD86 expression. H Macrophages were treated with $1 \mu \mathrm{g} / \mathrm{mL}$ FITC-labeled LPS (green) for $12 \mathrm{~h}$ and intracellular fluorescence was analyzed using fluorescence microscopy. DAPI nuclear staining was used to label the macrophage (blue), efferocytosis was observed via the number of LPS (green) containing in macrophages (blue).

the numbers of the recruited macrophages in the lymph nodes, suggesting that RvD1 could accelerate the removal of some of the recruited macrophages through the lymph. Previous studies show that inflammatory macrophages in the lungs, muscles, and nervous system are eliminated by apoptosis [18, 23, 24]. Two apoptotic pathways, namely the intrinsic and the extrinsic

pathways, have been identified in mammalian systems [25]. In the intrinsic apoptotic pathway, anti-apoptotic Bcl-2, Mcl-1, or proapoptotic Bax regulates mitochondrial membrane permeability [26]. In the extrinsic apoptotic pathway, the FasL on T cells binds to the Fas receptors on the targeted cells to accelerate apoptosis.
We found that treatment with RvD1 not only increased the level of Fas receptors and Fas mRNA on the recruited macrophages but also promoted the expression of FasL in $\mathrm{CD}^{+} \mathrm{T}$ cells in the resolution phase. The increased apoptosis of recruited macrophages was abrogated by treatment with Fas-blocking antibody. Treatment with RvD1 reduced $\mathrm{Mcl}-1$ expression in the recruited macrophages; however, RvD1 administration did not change the expression of Bax in vivo. We also found that the apoptosis of BMDMs peaked at $6-12 \mathrm{~h}$ after stimulation with LPS $(1 \mu \mathrm{g} / \mathrm{mL})$. BMDMs were co-cultured with LPS and RvD1 for $12 \mathrm{~h}$, and RvD1 was found to upregulate the expression of Fas receptors, but not $\mathrm{Mcl}-1, \mathrm{BCl}-2$, or Bax. Taken together, these results suggest that 
A

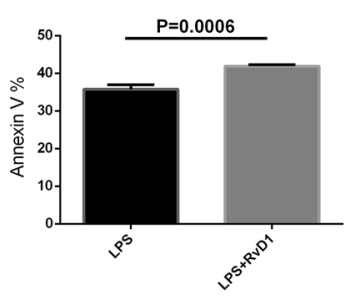

E

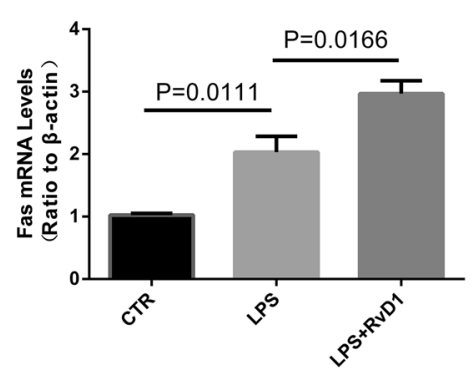

H

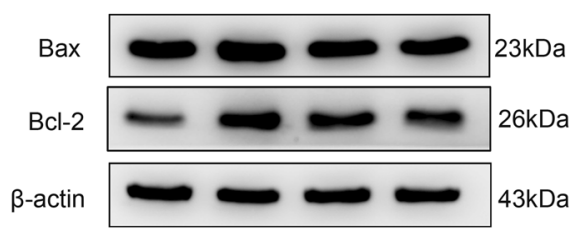

C
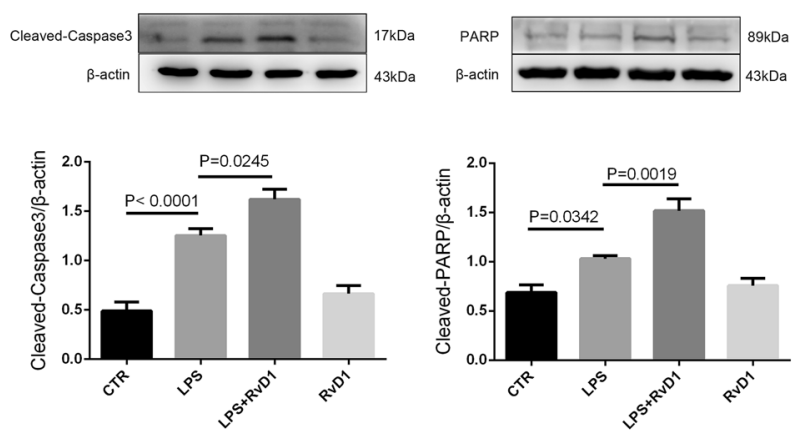

$\mathbf{F}$

G
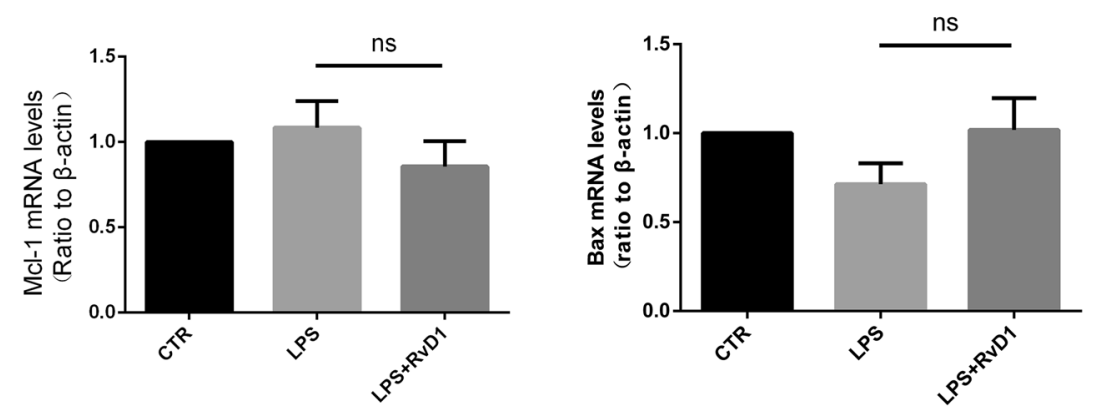

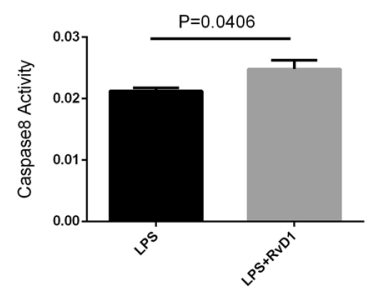

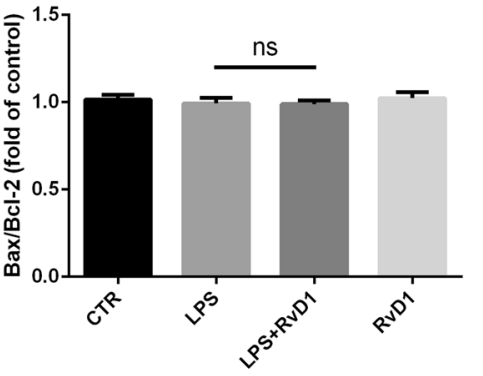

Fig. 6 RvD1 promotes apoptosis of BMDMs through Fas. A RvD1 promotes LPS-induced apoptosis in BMDMs. B Caspase 8 activity was also measured in BMDMs. Expression of cleaved caspase-3 (C) and cleaved PARP (D) was measured using western blotting. mRNA expression of Fas (E), Mcl-1 (F), and Bax (G) was measured using qPCR. H Western blot was used to confirm that RvD1 has no effect on Bax/Bcl-2.

RvD1 promoted apoptosis of the recruited macrophages mainly via the FasL-Fas pathway.

Caspases are a group of evolutionarily conserved cysteine proteins, functionally subdivided into initiators (caspase 8) and effectors (caspase-3) [27]. Fas signaling results in the formation of a death-inducing signal complex, which leads to the Fasassociated recruitment of caspase-8 through the adaptor protein of the death domain (FADD). Dimerization of caspase- 8 triggers activation of the apoptotic cascade activation effector, caspase-3 [28]. The DNA repair enzyme poly (ADP-ribose) polymerase (PARP), also called poly (ADP-ribose) synthetase or poly (ADP-ribose) transferase (PADRT), is a ribozyme activated by the DNA chain for participation in DNA repair. PARP cleavage by caspase- 3 is the defining feature of apoptosis [29]. Some special pro-inflammatory mediators, such as RvE1, have been shown to increase the activity of caspase- 8 and caspase-3, and promote phagocytosis-induced apoptosis [30]. We found that RvD1 could increase the activity of caspase-8, upregulate the levels of cleaved caspase- 3 and cleaved PARP, which indicated that this compound could promote apoptosis of the recruited macrophages partly through the FasLFas/caspase-3 signaling pathway.

ALX was the first receptor being identified. We found that the protective effect of RvD1 on lung tissues was abolished when the ALX inhibitor, BOC-2, was used. The RvD1-mediated reduction in neutrophil numbers was abrogated by BOC-2, and RvD1 treatment resulted in the upregulation of apoptosis. The expression of Fas and FasL on the recruited macrophages and $\mathrm{CD}^{+} \mathrm{T}$ cells, respectively, were also abolished by $\mathrm{BOC}-2$, indicating that the response of RvD1 was ALX dependent.

To summarize, our findings indicated that RvD1 enhanced efferocytosis and M2 phenotype in the recruited macrophages and reduced the inflammatory cytokines to alleviate LPS-induced tissue damage in the resolution phase. RvD1 also accelerated the removal of the recruited macrophages in the resolution phase mainly by apoptosis. Moreover, RvD1 promoted apoptosis of the recruited macrophages partly via the ALX/FasL-FasR/caspase3 signaling pathway (Fig. 8). Our findings reinforce the concept of therapeutic targeting of apoptosis in the recruited 
A

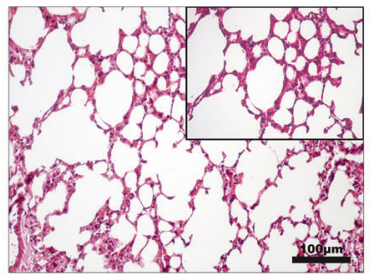

CTR

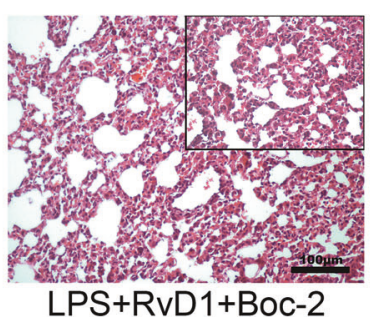

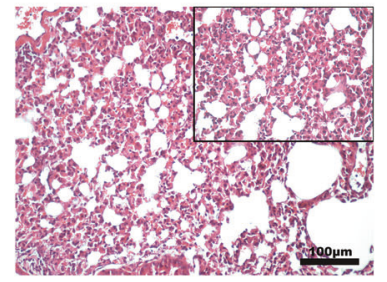

LPS

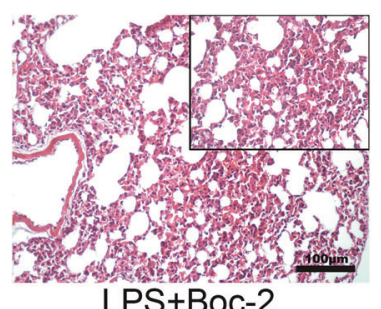

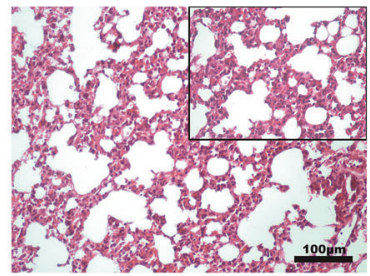

LPS+RvD1

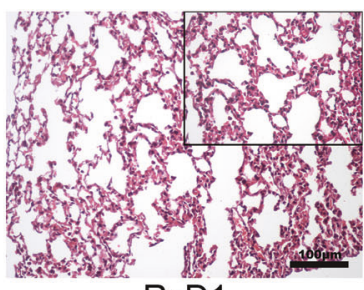

RvD1
B

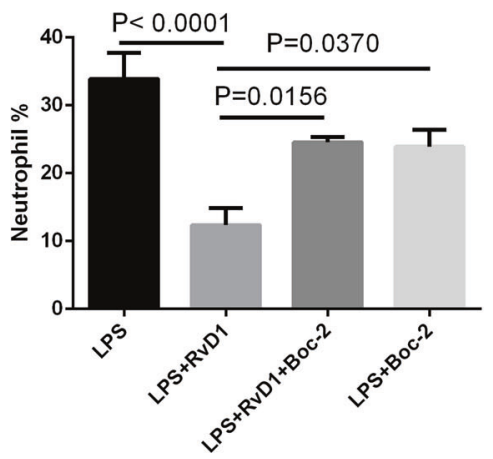

D

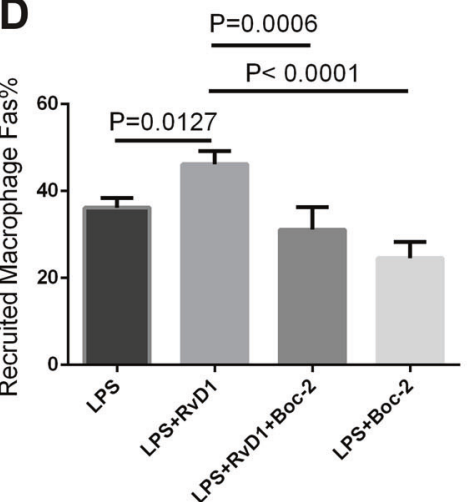

C

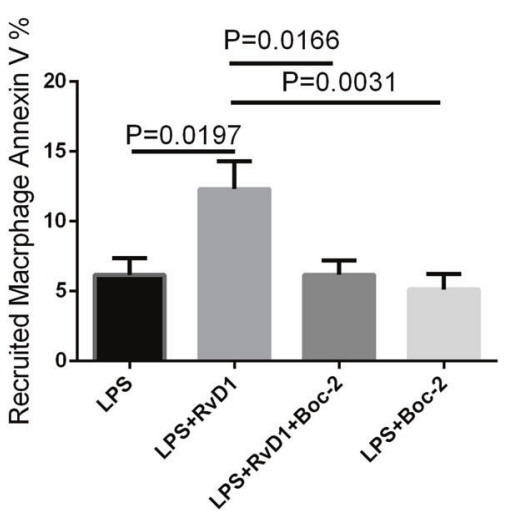

E

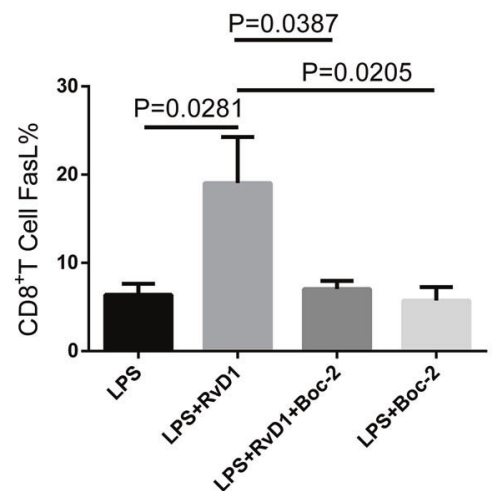

Fig. 7 The protective effect of RvD1 on lung tissues is dependent on ALX/PRP2. A The pro-resolution effect of RvD1 on lung injury was abrogated by the ALX/PRP inhibitor. B Percentage of neutrophils in BALF. C Percentage of annexin V-positive (apoptotic) recruited macrophages. D Percentage of Fas on recruited macrophages and E FasL expression on CD8 ${ }^{+}$T cells were measured using flow cytometry.

macrophages and suggest that RvD1 may provide a new therapy in the management of ALI/ARDS.

\section{MATERIALS AND METHODS \\ Reagents}

LPS (Escherichia coli serotype 055:B5) was obtained from Sigma (St. Louis, MO). RvD1 was obtained from Cayman Chemical Company (Ann Arbor, MI). F4/80-PE-Cyanine7, Ly-6G-FITC, CD206-PE, CD86-PE, CD3-APC, and CD8PerCy5.5 antibodies were procured from Invitrogen (Carlsbad, CA). FasL-PE and Fas-FITC were purchased from Biolegend (San Diego, CA) and the annexin V-PE/7-AAD apoptosis kit was purchased from MULTI SCIENCES. TNF- $\alpha$, IL-1 $\beta$, and IL- 6 ELISA kits were obtained from R\&D Systems
(Minneapolis, MN). Rabbit antimouse iNOS antibody, anti-Bax (E63), anticaspase- 3 , anti-BCl-2, and mouse antimouse $\beta$-actin were procured from Abcam (Cambridge, MA). The Fas-blocking antibody, antimouse CD178 monoclonal antibody (clone MFL3) was procured from eBioscience (Rockford, IL).

\section{Animal studies}

Specific pathogen-free adult C57BL/6 mice (female and male), each weighing $20-25 \mathrm{~g}$, were obtained from Charles River Laboratories. The mice were housed under controlled temperature and humidity conditions on a day-night cycle and were provided access to food and water ad libitum. All procedures were conducted in accordance with the Guide for 


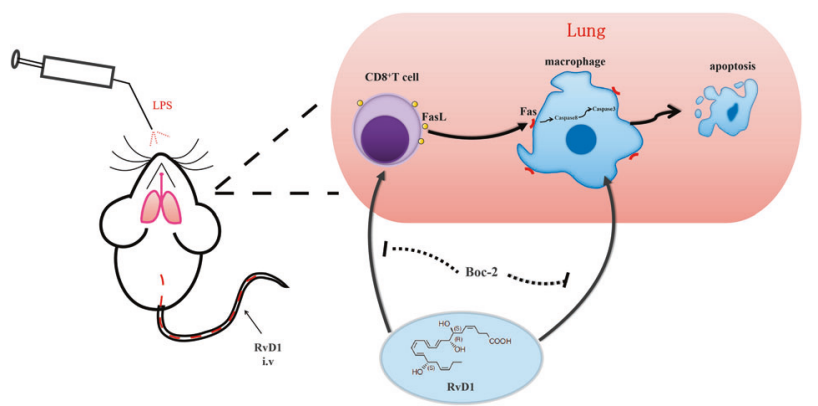

Fig. 8 RvD1 accelerates the resolution of inflammation by promoting apoptosis of the recruited macrophages. RvD1 promoted apoptosis of the recruited macrophages in the resolution phase partly via the ALX/FasL-FasR/caspase-3 signaling pathway.

the Care and Use of Laboratory Animals. This study was approved by the Animal Studies Ethics Committee of Wenzhou Medical University.

Rats were randomized into each group. Atomization inhalation of LPS ( $1 \mathrm{mg} / \mathrm{kg}$ ) was used to establish the lung injury mouse model. In the RvD1 group, the mice were administered RvD1 (100 ng/mouse) intravenously via the tail vein. In the LPS + RvD1 group, the mice were injected RvD1 intravenously via the tail vein 3 days after LPS stimulation, and the bronchoalveolar lavage fluid (BALF) was collected on day 5. For the Fasblocking experiments, the Fas-blocking antibody ( $30 \mathrm{mg}$ in $50 \mathrm{ml}$ PBS) was administered intratracheally on days 3 and 4 after LPS, BALF was collected on day 5.

All the mice were included. For each group used in experiments, at least six mice were guaranteed for adequate power to detect a pre-specified effect size. Animal studies were blinded during the group allocation, experiments, and when assessing the outcomes.

\section{ELISA}

TNF- $\alpha$, IL-1 $\beta$, and IL-6 levels in the lung tissue homogenate were measured using the respective ELISA kits according to manufacturers' protocol.

\section{Cell culture}

Bone marrow-derived macrophages (BMDMs) were isolated from 6-weekold BALB/C mice and their concentration was adjusted to $2 \times 10^{6}$ cells $/ \mathrm{mL}$ using RPMI1640 medium. The cells were plated in a $10-\mathrm{cm}$ culture dish and were allowed to differentiate in a humidified incubator in an atmosphere of $5 \% \mathrm{CO}_{2}$ at $37^{\circ} \mathrm{C}$. The medium was changed every 2-3 days. After 7 days, BMDMs $\left(1 \times 10^{6}\right.$ cells $\left./ \mathrm{mL}\right)$ were plated in 6-well plates. Cells were divided into four groups, namely, control, LPS, LPS + RvD1, and RvD1. All cells were cultured with LPS $(1 \mu \mathrm{g} / \mathrm{mL})$ in the presence or absence of RvD1 (100 nM) for $12 \mathrm{~h}$, and efferocytosis and apoptosis were measured.

\section{Flow cytometry}

BALF cells were collected and immediately treated with antimouse CD16/ 32 antibody (BD) for 20 min to block nonspecific binding. Macrophages were incubated with monoclonal antibodies directed at F4/80 (PE-Cy7), Ly6C (APC), and Fas (FITC). T lymphocytes were stained with a monoclonal antibody directed at CD3 (APC), CD4 (FITC), CD8 (PerCy5.5), FasL-PE, and analyzed using flow cytometry.

\section{Quantitative real-time RT-PCR}

Recruited macrophages from the BALF were sorted using a CytoFLEX flow cytometer (Beckman Coulter). The total RNA of the recruited macrophages was isolated using a miRNeasy Mini Kit (Qiagen) according to the manufacturer's protocol. The cDNA was synthesized using a reverse transcription kit (Thermo). Gene expression was detected using TB Green Premix Ex Taq $<$ (Takara). Samples were normalized to the mRNA expression of the housekeeping gene ( $\beta$-actin to analyze samples from mice) and the results are expressed as fold increase using the $2^{-\Delta \Delta C t}$ method. The primer pairs of each gene were as follows:

Fas:5'-GCGGGTTCGTGAAACTGATAA-3', 5'-GCAAAATGGGCCTCCTIGATA3'; FasL:5'-GGAGTGGTCCTTAATGCCT-3', $5^{\prime}$-TCTTTCTCTGTGCCTCTGC-3';

MCL-1:5'-GACGAAACGGGAACTGGCTTGTC-3', 5'-GCCGCGTTGTAGGTCGTGTAC-3'; BAX:5'-AGACAGGGGCCTITITGCTAC-3'， 5'-AATTCGCCGGAGACACTC
G-3'; $\quad \beta$-actin: $\quad 5^{\prime}$-GGCTGTATTCCCCTCCATCG-3', $5^{\prime}$-CCAGTTGGTAACAATGCCATGT-3'.

\section{Western blotting}

Cell extracts were prepared by suspending the cells directly in the RIPA lysis buffer and PMSF for $15 \mathrm{~min}$ on ice, followed by centrifugation for $25 \mathrm{~min}$ at $12,000 \times g$. The supernatant was collected and protein concentration was measured using a protein assay kit. Protein samples $(30 \mu \mathrm{g})$ were separated using SDS-PAGE and transferred to nitrocellulose membranes. Nonspecific protein binding was blocked using $10 \%$ non-fat dry milk-PBST buffer for $1 \mathrm{~h}$ at room temperature. The blots were then incubated overnight with the primary antibodies against iNOS, Bax, and $\mathrm{BCl}-2$ at 1:1000 dilutions. Then, they were washed three times with PBST and incubated for $1 \mathrm{~h}$ with the appropriate secondary antibodies coupled to horseradish peroxidase at 1:2000 dilutions. The protein bands were quantified using a UVP gel imaging system.

\section{Phagocytosis assay}

To analyze the percentage of macrophages phagocytosis in vivo, FITC-LPS was administered via tracheal inhalation and RvD1 was injected through the tail vein. Twelve hours later, the percentage of FITC-LPS in the recruited macrophages was detected using flow cytometry. For in vitro experiments, BMDMs were cultured with FITC-LPS in the presence or absence of RvD1 for $12 \mathrm{~h}$, and phagocytosis was determined using fluorescence microscopy.

\section{Statistical analysis}

Data are presented as the mean \pm SEM, and numbers of experiments $(n)$ are indicated. Statistical tests are justified as appropriate for every figure, and the data meet the assumptions of the tests, including normal distribution. The estimated variance is similar between the groups that are being statistically compared. Data were analyzed using one-way ANOVA, Tukey test was used for posthoc comparison. A value of $p<0.05$ was considered statistically significant. Statistical analyses were performed using SPSS 19.0 and Prism 6.0.

\section{DATA AVAILABILITY}

The datasets used and/or analyzed during the current study are available from the corresponding author on reasonable request.

\section{REFERENCES}

1. Matthay MA, McAuley DF, Ware LB. Clinical trials in acute respiratory distress syndrome: challenges and opportunities. Lancet Respir Med. 2017;5:524-34.

2. Wheeler AP, Bernard GR. Acute lung injury and the acute respiratory distress syndrome: a clinical review. Lancet. 2007;369:1553-64.

3. Lomas-Neira J, Chung CS, Perl M, Gregory S, Biffl W, Ayala A. Role of alveolar macrophage and migrating neutrophils in hemorrhage-induced priming for ALI subsequent to septic challenge. Am J Physiol Lung Cell Mol Physiol. 2006;290:51-8.

4. Gibbings SL, Goyal R, Desch AN, Leach SM, Prabagar M, Atif SM, et al. Transcriptome analysis highlights the conserved difference between embryonic and postnatal-derived alveolar macrophages. Blood. 2015;126:1357-66.

5. Aegerter H, Kulikauskaite J, Crotta S, Patel H, Kelly G, Hessel EM, et al. Influenzainduced monocyte-derived alveolar macrophages confer prolonged antibacterial protection. Nat Immunol. 2020;21:145-57.

6. Basil MC, Levy BD. Specialized pro-resolving mediators: endogenous regulators of infection and inflammation. Nat Rev Immunol. 2016;16:51-67.

7. Wang B, Gong X, Wan JY, Zhang L, Zhang Z, Li HZ, et al. Resolvin D1 protects mice from LPS-induced acute lung injury. Pulm Pharm Ther. 2011;24:434-41.

8. Sun Y-P, Oh SF, Uddin J, Yang R, Gotlinger K, Campbell E, et al. Resolvin D1 and Its Aspirin-triggered 17R Epimer. Stereochemical assignments, anti-inflammatory properties, and enzymatic inactivation. J Biol Chem. 2007;282:9323-34.

9. Duffield JS, Hong S, Vaidya VS, Lu Y, Fredman G, Serhan CN, et al. Resolvin D series and protectin D1 mitigate acute kidney injury. J Immunol. 2006;177:5902-11.

10. Dalli J, Serhan CN. Specific lipid mediator signatures of human phagocytes: microparticles stimulate macrophage efferocytosis and pro-resolving mediators. Blood. 2012;120:60-72.

11. Gordon S, Taylor PR. Monocyte and macrophage heterogeneity. Nat Rev Immunol. 2005;5:953-64. 
12. Hashimoto D, Chow A, Noizat C, Teo P, Beasley MB, Leboeuf M, et al. Tissueresident macrophages self-maintain locally throughout adult life with minimal contribution from circulating monocytes. Immunity. 2013;38:792-804.

13. Johnston LK, Rims CR, Gill SE, McGuire JK, Manicone AM. Pulmonary macrophage subpopulations in the induction and resolution of acute lung injury. Am J Respir Cell Mol Biol. 2012;47:417-26.

14. Amano $H$, Morimoto $K$, Senba $M$, Wang $H$, Ishida $Y$, Kumatori $A$, et al. Essential contribution of monocyte chemoattractant protein-1/C-C chemokine ligand- 2 to resolution and repair processes in acute bacterial pneumonia. J Immunol. 2004;172:398-409.

15. Zasłona Z, Przybranowski S, Wilke C, van Rooijen N, Teitz-Tennenbaum S, Osterholzer JJ, et al. Resident alveolar macrophages suppress, whereas recruited monocytes promote, allergic lung inflammation in murine models of asthma. J Immunol. 2014;193:4245-53.

16. Duffield JS, Forbes SJ, Constandinou CM, Clay S, Partolina M, Vuthoori S, et al. Selective depletion of macrophages reveals distinct, opposing roles during liver injury and repair. J Clin Inves. 2005;115:56-65.

17. Thepen T, van Vuuren AJ, Kiekens RC, Damen CA, Vooijs WC, van De Winkel JG. Resolution of cutaneous inflammation after local elimination of macrophages. Nat Biotechnol. 2000;18:48-51.

18. Janssen WJ, Barthel L, Muldrow A, Oberley-Deegan RE, Kearns MT, Jakubzick C, et al. Fas determines differential fates of resident and recruited macrophages during resolution of acute lung injury. Am J Respir Crit Care Med. 2011;184:547-60.

19. Kearns MT, Barthel L, Bednarek JM, Yunt ZX, Henson PM, Janssen WJ. Fas ligandexpressing lymphocytes enhance alveolar macrophage apoptosis in the resolution of acute pulmonary inflammation. Am J Physiol Lung Cell Mol Physiol. 2014;307:L62-70.

20. Matthay MA, Zemans RL, Zimmerman GA, Arabi YM, Beitler JR, Mercat A, et al. Acute respiratory distress syndrome. Nat Rev Dis Prim. 2019;5:18.

21. Titos E, Rius B, González-Périz A, López-Vicario C, Morán-Salvador E, MartínezClemente $M$, et al. Resolvin D1 and its precursor docosahexaenoic acid promote resolution of adipose tissue inflammation by eliciting macrophage polarization toward an M2-like phenotype. J Immunol. 2011;187:5408-18.

22. Kang JW, Lee SM. Resolvin D1 protects the liver from ischemia/reperfusion injury by enhancing M2 macrophage polarization and efferocytosis. Biochim Biophys Acta. 2016;1861:1025-35.

23. Kuhlmann T, Bitsch A, Stadelmann C, Siebert H, Brück W. Macrophages are eliminated from the injured peripheral nerve via local apoptosis and circulation to regional lymph nodes and the spleen. J Neurosci. 2001;21:3401-8.

24. Tidball JG, St Pierre BA. Apoptosis of macrophages during the resulution of muscle inflammation. J Leukoc Biol. 1996;59:380-8.

25. Riedl SJ, Salvesen GS. The apoptosome: signalling platform of cell death. Nat Rev Mol Cell Biol. 2007;8:405-13.

26. Ghobrial IM, Witzig TE, Adjei AA. Targeting apoptosis pathways in cancer therapy. CA Cancer J Clin. 2005;55:178-94.

27. Van Opdenbosch N, Lamkanfi M. Caspases in Cell Death, Inflammation, and Disease. Immunity. 2019;50:1352-64.

28. Man SM, Kanneganti TD. Converging roles of caspases in inflammasome activation, cell death and innate immunity. Nat Rev Immunol. 2016;16:7-21.

29. Pieper AA, Verma A, Zhang J, Snyder SH. Poly (ADP-ribose) polymerase, nitric oxide and cell death. Trends Pharm Sci. 1999;20:171-81.

30. El Kebir D, Gjorstrup P, Filep JG. Resolvin E1 promotes phagocytosis-induced neutrophil apoptosis and accelerates resolution of pulmonary inflammation. Proc Natl Acad Sci USA. 2012;109:14983-8.

\section{ACKNOWLEDGEMENTS}

We are grateful to the editors, the reviewers and our colleagues for their valuable comments and efforts. This work was sponsored by grants from the National Natural Science Foundation of China (no. 81570076) and by the Natural Science Foundation of Zhejiang Province (LY18H010005), Provincial Medical and health science and technology project (2021455293), Wenzhou Science and Technology Bureau Project (No.Y20210090), Clinical Research Fundation of the 2nd Affiliated Hospital of Wenzhou Medical University (SAHoWMU-CR2018-11-134) and Research Fund for Lin He's Academician Workstation of New Medicine and Clinical Translation (19331102).

\section{AUTHOR CONTRIBUTIONS}

X.S.Y., Y.Y., and Y.Q. performed the experiments and writing of the paper; X.H.R. and S.C.X. provided acquisition, analysis and interpretation of data, and statistical analysis; X.S.Y., Y.Y., M.M.Q., and J.S.W. review and revision of the paper; M.H.X., Z.S.X., and F.G. S. provided technical support; J.S.W. and W.Q. performed study concept and design. All authors read and approved the final paper.

\section{ETHICS APPROVAL}

All experiments were approved by the Ethics Committee of Wenzhou Medical University.

\section{COMPETING INTERESTS}

The authors declare no competing interests.

\section{ADDITIONAL INFORMATION}

Correspondence and requests for materials should be addressed to Sheng-wei Jin or Qian Wang.

Reprints and permission information is available at http://www.nature.com/ reprints

Publisher's note Springer Nature remains neutral with regard to jurisdictional claims in published maps and institutional affiliations.

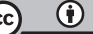

Open Access This article is licensed under a Creative Commons Attribution 4.0 International License, which permits use, sharing, adaptation, distribution and reproduction in any medium or format, as long as you give appropriate credit to the original author(s) and the source, provide a link to the Creative Commons license, and indicate if changes were made. The images or other third party material in this article are included in the article's Creative Commons license, unless indicated otherwise in a credit line to the material. If material is not included in the article's Creative Commons license and your intended use is not permitted by statutory regulation or exceeds the permitted use, you will need to obtain permission directly from the copyright holder. To view a copy of this license, visit http://creativecommons. org/licenses/by/4.0/.

(c) The Author(s) 2021 